\title{
Silencing of GRP94 expression promotes apoptosis in pancreatic cancer cells
}

\author{
ZHENG PAN, MERT ERKAN, SYLVIA STREIT, HELMUT FRIESS and JÖRG KLEEFF \\ Department of General Surgery, Technische Universität München, Munich, Germany
}

Received April 22, 2009; Accepted June 17, 2009

DOI: 10.3892/ijo_00000395

\begin{abstract}
As a molecular chaperone, GRP94 is the most abundant glycoprotein in the endoplasmic reticulum, playing an important role in maintaining cellular homeostasis. Here, we investigated the expression and the role of GRP94 in regulating cell growth and apoptosis in pancreatic cancer cells. GRP94 mRNA levels were analyzed by QRT-PCR. Immunohistochemistry was performed to localize GRP94 in tissues of the normal pancreas $(n=20)$, chronic pancreatitis $(n=20)$ and pancreatic ductal adenocarcinoma $(n=44)$. Silencing of GRP94 expression was carried out by transfection with specific siRNA oligonucleotides. Apoptosis was induced by treatment with actinomycin D. Compared to normal pancreatic tissues, median mRNA levels of GRP94 were 1.5- and 3.7-fold $(\mathrm{p}<0.05)$ lower in chronic pancreatitis and pancreatic cancer tissues, respectively. GRP94 protein was strongly expressed in normal acinar cells and moderately expressed in normal ductal cells. GRP94 expression was lost in $48 \%$ of the cancer cases. Moderate or strong staining in cancer cells was observed in 32 and $20 \%$ of pancreatic cancer tissues, respectively. Silencing GRP94 by siRNA increased apoptosis of pancreatic cancer cells in vitro. Patients with higher than the median expression have a tendency for a worsened survival. When the small number of patients with the highest expression $(n=3)$ were compared with the rest of the group $(n=41)$, the survival difference was significantly worse (5 vs. 18 months, respectively, $\mathrm{p}=0.006$ ). Downregulation of GRP94 decreases apoptosis resistance in pancreatic cancer cells. Clinically, patients with high GRP94 expression show a tendency for a worsened survival.
\end{abstract}

\section{Introduction}

Nearly $90 \%$ of pancreatic neoplasms are ductal adenocarcinomas (1). These are characterized by tumor desmoplasia, early local extension to contiguous structures, metastases to

Correspondence to: Dr Jörg Kleeff, Department of General Surgery, Technische Universität München, Klinikum rechts der Isar, D-81675 Munich, Germany

E-mail: kleeff@gmx.de

Key words: TRA1, chemotherapy, apoptosis, capheron, pancreatic cancer regional lymph nodes and to the liver (2-4). Moreover, pancreatic cancer cells are usually resistant to the programmed cell death (apoptosis) mediated by conventional chemotherapeutic agents $(5,6)$. It is generally believed that cancer cells have an altered cellular physiology characterized by abundance of growth signals, insensitivity to cycle arrest signals, and evasion of apoptosis (7). Unresponsiveness to apoptotic stimuli can result in tumor progression and resistance to most oncologic therapies (5).

It has been established that the glucose-regulated proteins (GRPs) play an important role in maintaining cellular homeostasis. As endoplasmic reticulum (ER) chaperones, they participate in ER protein translocation, chaperoning, protein quality control, ER-associated protein degradation, ER stress sensing and regulation, and ER calcium binding (8). Recently, the ER stress pathways and the GRPs have been linked to cancer growth and drug resistance $(9,10)$. As such, they represent novel markers for cancer progression and chemoresponsiveness, as well as targets for cancer therapy. Some GRPs are also capable of modulating innate and adaptive immunity, and have been studied extensively in cancer immunotherapy (11-13).

GRP94 is the most abundant glycoprotein in the endoplasmic reticulum. GRP94 knockdown mouse embryos die in early gestation (14) and GRP94 is up-regulated in transformed cells, suggesting an expanded role for this chaperone beyond the maturation of immune response proteins. Overexpression of GRP94 is associated with tumorigenicity, and decreased sensitivity to radiation, whereas suppression of GRP94 sensitizes cells to etoposide treatment (15-18). GRP94, as in the case of the antiapoptotic protein $\mathrm{Bcl}-2$, can be a target of proteolytic cleavage itself during the apoptotic process (18). Overexpression of GRP94 suppresses ER stress-induced apoptosis of neuronal cells (19), whereas down-regulation of GRP94 expression accelerates ER stress-induced apoptosis $(18,19)$. A recent report has shown that GRP94 blocks apoptosis induced by HCV infection (20). These findings suggest that GRP94 protects against ER stress-induced apoptosis. Despite these observations, effects of GRP94 expression in pancreatic cancer have not been extensively investigated.

We have previously shown that actinomycin $\mathrm{D}$ is a potent inducer of apoptosis in a variety of pancreatic cancer cells in vitro (21). It binds to DNA and inhibits RNA and protein synthesis. Actinomycin D may act via JNK/SAPK and Bax to promote apoptosis in Panc-1 cells. Moreover, several lines 
of evidence indicate that actinomycin D causes apoptotic and not necrotic cell death (21).

In the present study, the expression of GRP94 was analyzed in the normal pancreas, pancreatic cancer, and chronic pancreatitis tissues. We also investigated the role of GRP94 in regulating cell growth and apoptosis in pancreatic cancer cells.

\section{Materials and methods}

Reagents. The following reagents were purchased: RPMI-1640, Dulbecco's modified Eagle's medium, Trypsin-EDTA, and penicillin-streptomycin from Invitrogen (Karlsruhe, Germany); fetal bovine serum from PAN Biotech (Aidenbach, Germany); GRP94 polyclonal rabbit antibody and GAPDH polyclonal rabbit antibody from Santa Cruz Biotechnology (Santa Cruz, CA); synthetic siRNA oligonucleotides for GRP94 and HiPerFect transfection reagent from Qiagen (Hilden, Germany); ß-actin rabbit antibody from BD Biosciences (Erembodegem, Belgium); actinomycin D from Sigma (Sigma, St. Louis, MO). ECL immunoblotting detection reagents from Amersham Biosciences (Amersham Life Science, Amersham, UK); Envision system, liquid $\mathrm{DAB}^{+}$ substrate and antibody diluent from Dako (Hamburg, Germany); mini protease inhibitor from Roche Molecular Biochemicals (Basel, Switzerland); BCA protein assay from Pierce Chemical Co. (Rockford, IL); and RNA-later solution from Ambion (Huntington, UK). All reagents and equipment for mRNA and cDNA preparation were purchased from Roche (Roche Applied Science, Mannheim, Germany).

Tissue specimens and cell cultures. Tissues of pancreatic ductal adenocarcinoma (PDAC) and chronic pancreatitis (CP) were collected at the University of Heidelberg, Germany. Normal human pancreatic tissue samples were obtained through an organ donor program from previously healthy individuals. All samples were confirmed histologically. Freshly removed tissues were fixed in paraformaldehyde solution for 12-24 $\mathrm{h}$ and then paraffin-embedded for histological analysis. In addition, a portion of the tissue samples was preserved in RNAlater (Ambion Europe Ltd., Huntingdon, Cambridgeshire, UK), or snap-frozen in liquid nitrogen immediately upon surgical removal and maintained at $-80^{\circ} \mathrm{C}$ until use. The Human Subjects Committee of the University of Heidelberg, Germany, approved the use of human tissues for molecular research. Written informed consent was obtained from all patients.

Pancreatic cancer cell lines were grown in DMEM medium (Panc-1 and MiaPaCa-2) or RPMI-1640 (Aspc-1, BxPc-3, Capan-1, Colo-357, SU86.86, and T3M4), supplemented with $10 \%$ fetal bovine serum (FBS) and $100 \mathrm{U} / \mathrm{ml}$ penicillin and streptomycin (complete medium), and incubated at $37^{\circ} \mathrm{C}$ in a $5 \% \mathrm{CO}_{2}$ humidified atmosphere.

Quantitative real-time PCR. RNA was reverse transcribed into cDNA using the cDNA synthesis kit for reverse transcription polymerase chain reaction (PCR) according to the manufacturer's instructions. Quantitative real-time PCR (QRT-PCR) was performed with the Light Cycler Fast Start DNA SYBR Green kit. The number of specific transcripts was normalized to the levels of the housekeeping gene HPRT. Specific primers were used: GRP94 forward 5'-TTGCCAGACCATCCGTA CTG-3'; GRP94 reverse 5'-GAATTGGATGAAAGATAAA GCCCTTA-3'.

Immunohistochemistry. Immunohistochemistry was performed using the Dako Envision System, as published previously (22). Briefly, consecutive paraffin-embedded tissue sections ( $3 \mu \mathrm{m}$ thick) were deparaffinized and rehydrated using routine methods. Antigen retrieval was performed by pretreatment of the slides in citrate buffer ( $\mathrm{pH}$ 6.0) in a microwave oven for $15 \mathrm{~min}$. Endogenous peroxidase activity was quenched by incubation in deionized water containing $3 \%$ hydrogen peroxide at room temperature for $10 \mathrm{~min}$. Sections were incubated with rabbit anti-human GRP94 polyclonal antibodies (1:250) diluted with antibody diluent at $4^{\circ} \mathrm{C}$ overnight and then incubated with horseradish peroxidase (HRP)conjugated anti-rabbit antibody and counterstaining with Mayer's hematoxylin. In addition, to confirm the specificity of the primary antibodies, tissue sections were incubated with negative control rabbit $\operatorname{IgG}$. Under these conditions, no specific immunostaining was detected.

Semi-quantitative analysis of GRP94 expression in tissues. Semiquantitative analysis was performed as published previously (22). Scores were given separately for the stained area and for the intensity of staining. Quantification was made as follows: $1,<33 \%$ of the cancer cell; $2,33-66 \%$ of the cells; $3,>66 \%$ of the cancer cells. Intensity of staining was quantified as follows: 1 , absent; 2 , weak/moderate; 3 , strong. Each section had a final grade that derived from the multiplication of the area and intensity scores. GRP94 expression was considered to be absent/low for grade 1, moderate for grades 2, 3, and 4, and strong for grades 6 and 9 .

Immunoblot analysis. Cultured pancreatic cancer cells were lysed in ice-cold buffer containing $20 \mathrm{mM}$ Tris- $\mathrm{HCl}$ (pH 7.4), $150 \mathrm{mM} \mathrm{NaCl}, 0.1 \%$ TritonX-100, $2.5 \mathrm{mM}$ sodium pyrophosphate, and 1 tablet EDTA-free protease inhibitor cocktail for $30 \mathrm{~min}$.

Cell lysates were then collected after centrifugation at $13500 \mathrm{rpm}$ for $10 \mathrm{~min}$ at $4^{\circ} \mathrm{C}$. Total protein $(20 \mu \mathrm{g})$ was loaded on $10 \%$ polyacrylamide gels and transferred to nitrocellulose membranes. Membranes were blocked in $20 \mathrm{ml}$ TBS, 5\% skim milk and $0.05 \%$ Tween-20 for $1 \mathrm{~h}$ and incubated with rabbit anti-GRP94 polyclonal antibody (1:400) overnight at $4^{\circ} \mathrm{C}$. Membranes were washed three times with $0.05 \%$ Tween-20-TBS and incubated with horseradish peroxidase (HRP)-conjugated anti-rabbit antibody (1:5000) for $2 \mathrm{~min}$ at room temperature. Signals were detected using the enhanced chemiluminescence system (ECL, Amersham Life Science Ltd., Bucks, UK). GAPDH (1:5000) was used to verify equal loading.

siRNA transfection. Synthetic siRNA oligonucleotides for GRP94 were prepared and stored according to the manufacturer's instructions. The target sequences are 5'-TCGCCT CAGTTTGAACATTGA-3'; 5'-AAGTTGATGTGGATG GTACAT-3'. Cells were grown until $70 \%$ confluence and transfections were carried out with HiPerFect trqansfection reagent according to the manufacturer's instructions. The 


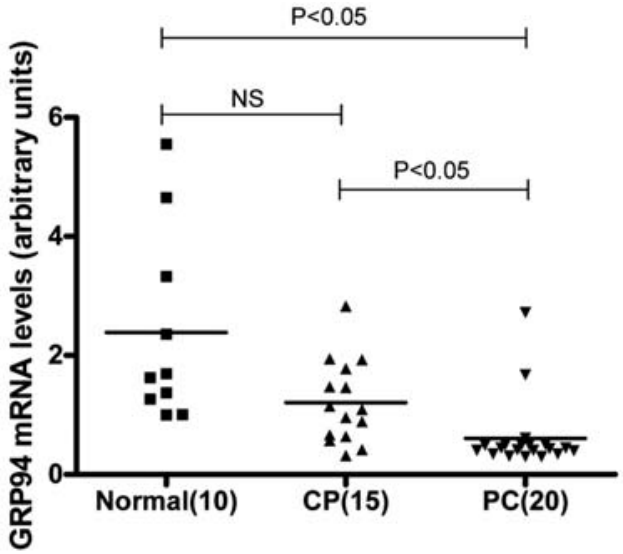

Figure 1. GRP94 mRNA expression in pancreatic tissues. Real-time quantitative RT-PCR analysis of GRP94 mRNA levels in normal pancreas, chronic pancreatitis, and pancreatic cancer tissues was carried out as described in Materials and methods. RNA input was normalized to the average expression of the housekeeping gene HPRT. Horizontal lines represent the median values.

final concentration of both the control and specific oligonucleotides was $5 \mathrm{nM}$. The efficacy of the siRNA transfection was ascertained by immunoblot analysis after 48-120 h of transfection.

Cell growth assay. Cell growth was determined using the 3-(4,5-methylthiazol-2-yl)-2,5-diphenyltetrazolium bromide (MTT) colorimetric growth assay. Cells were seeded at a density of 5000 cells/well in 96-well plates for up to five days. All assays were performed in triplicate and repeated four times.
Actinomycin D treatment. Transfected cells $(5,000 /$ well $)$ were seeded overnight in 96-well plates and incubated for $48 \mathrm{~h}$ under increasing concentrations of actinomycin D. Cell growth was assessed by the MTT assay, as published previously (5). All assays were performed in triplicate and repeated four times.

Statistical analysis. Statistical analysis and graph presentation were carried out using the GraphPad Prism 4 Software (GraphPad, San Diego, CA). mRNA results of QRT-PCR analyses are presented as mean \pm SEM. The Shapiro-Wilk test was used to evaluate data distribution. The Mann-Whitney test was used for comparison of two groups with independent samples. One-way analysis of variance (ANOVA) and Bonferroni's multiple comparison tests were used to compare three groups. The Kaplan-Meier method and log-rank test were used for survival analysis. $\mathrm{P}<0.05$ was taken as the level of significance.

\section{Results}

Expression of GRP94 in pancreatic tissues. To quantify the mRNA levels of GRP94, QRT-PCR was performed using tissues of the normal pancreas $(n=10)$, chronic pancreatitis $(n=15)$ and pancreatic ductal adenocarcinoma $(n=20)$. Compared to normal pancreatic tissues, median mRNA levels of GRP94 were 1.5- and 3.7-fold $(\mathrm{p}<0.05)$ lower in chronic pancreatitis and pancreatic cancer tissues, respectively (Fig. 1). Importantly, chronic pancreatitis tissues also had 2.5-fold $(\mathrm{p}<0.05)$ higher GRP94 mRNA than pancreatic cancer tissues.
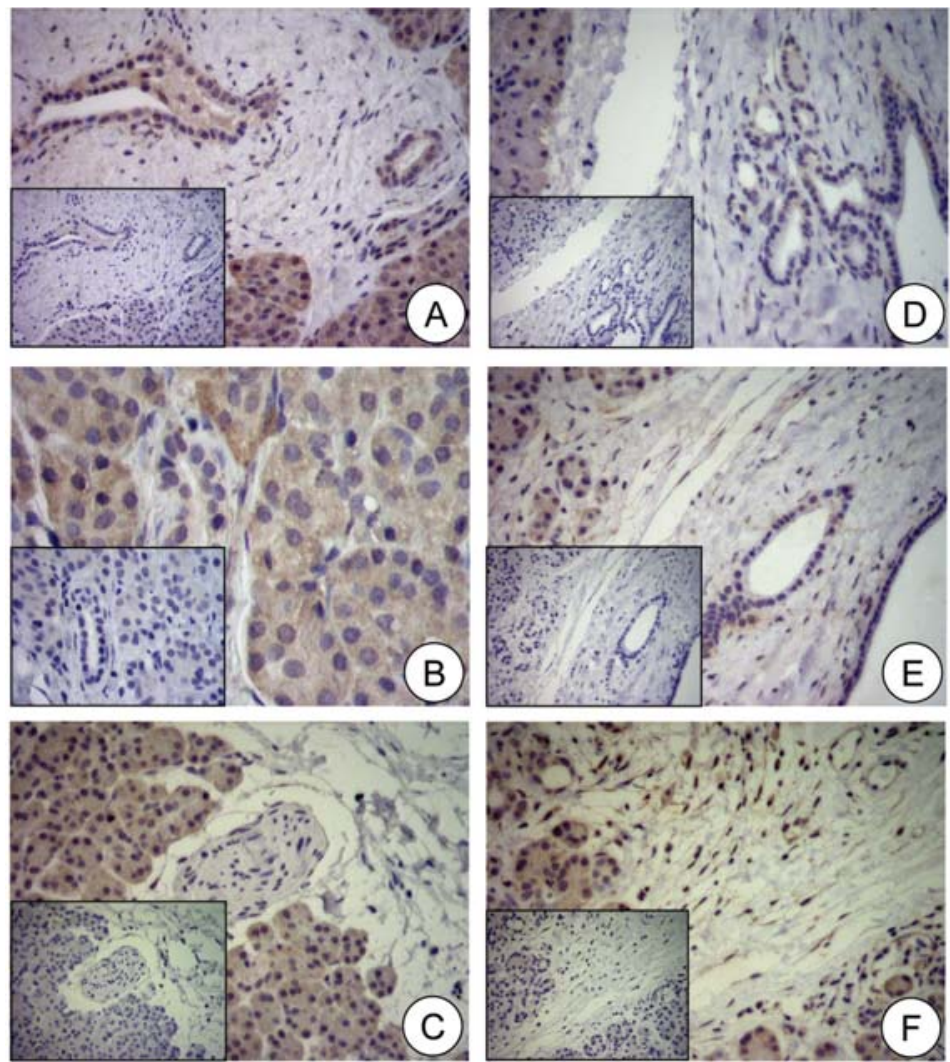
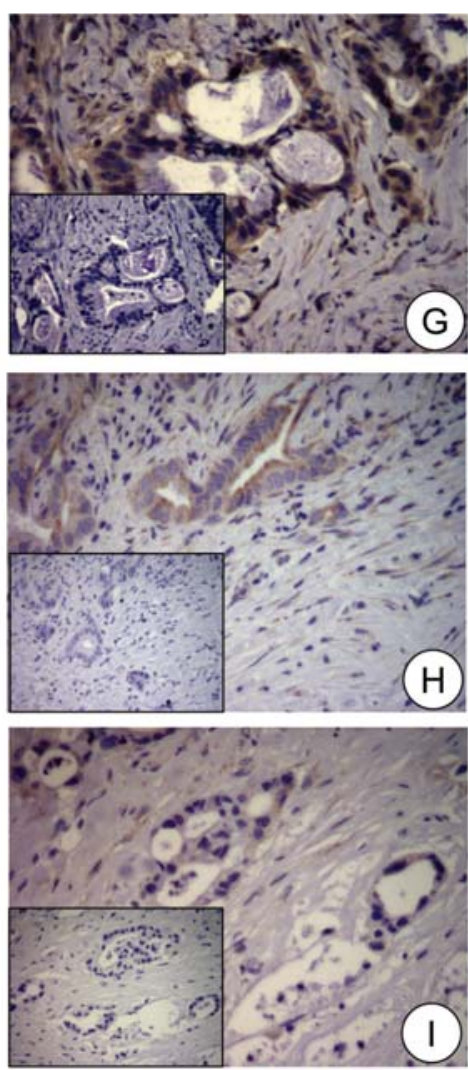

Figure 2. Localization of GRP94 in pancreatic tissues. Immunohistochemistry using a specific GRP94 antibody was carried out as described in Materials and methods. GRP94 expression in the normal pancreas (A-C), chronic pancreatitis (D-F) and pancreatic ductal adenocarcinoma (G- I) is shown. A-I-insets, negative control sections using isotype-matched $\mathrm{IgG}$ are shown. 

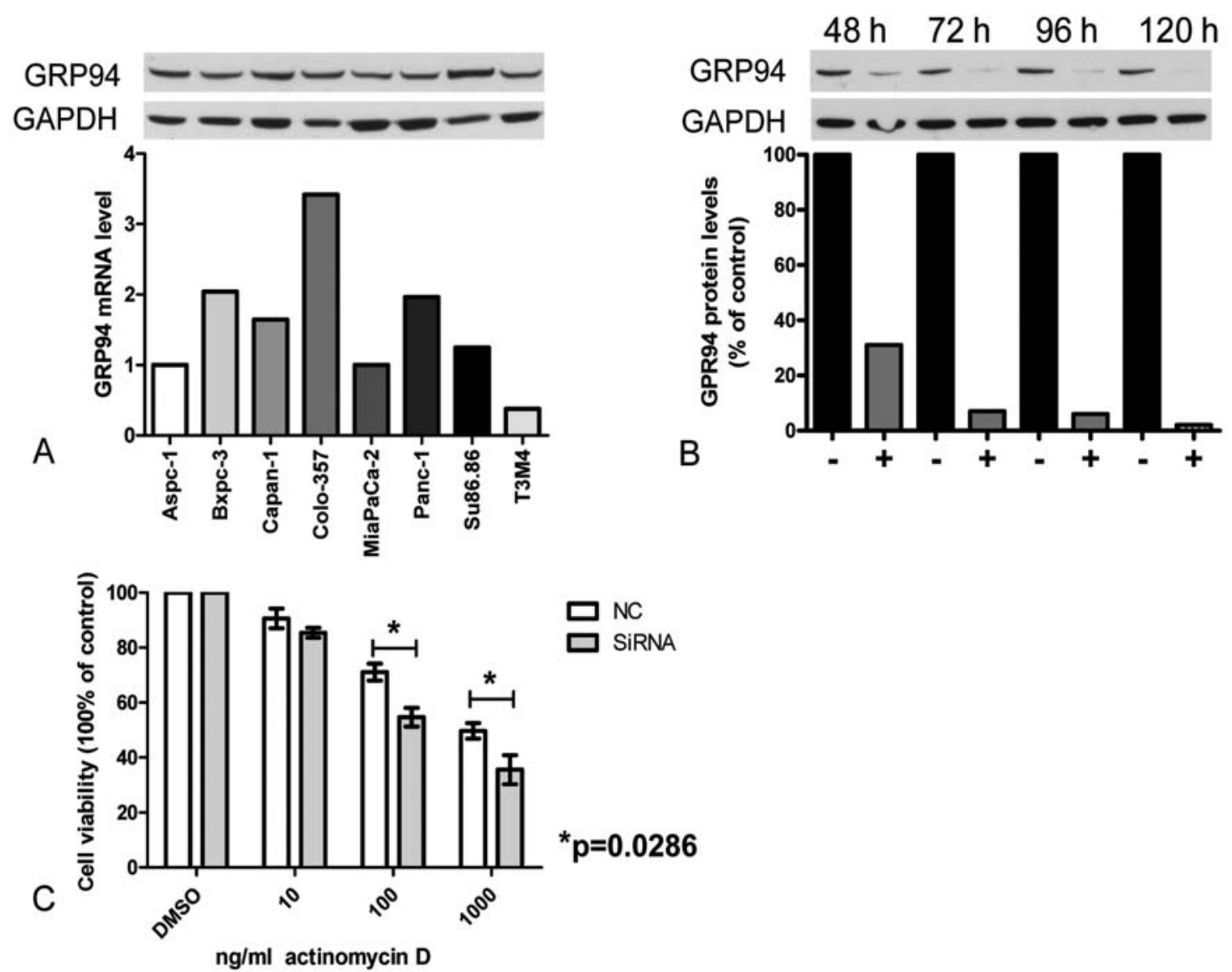

Figure 3. Expression of GRP94 in pancreatic ductal adenocarcinoma cell lines and the effect of silencing of GRP94 in Panc-1 on apoptosis resistance. (A) Realtime quantitative RT-PCR analysis of GRP94 mRNA levels in 8 pancreatic cancer cell lines, as described in Materials and methods. RNA input was normalized to the average expression of the housekeeping gene HPRT. Immunoblot analysis was carried out to detect expression of GRP94 in eight pancreatic cancer cell lines. Equal loading of the protein samples was confirmed using a GAPDH antibody. (B) Expression of GRP94 after transfection with GRP94 siRNA (+) and siRNA control (-) at different time-points in Panc-1 cells. Equal loading of the protein samples was confirmed using a GAPDH antibody. (C) Cells were incubated for $24 \mathrm{~h}$ in the absence (DMSO was used as control) or presence of 10, 100 and $1000 \mathrm{ng} / \mathrm{ml}$ actinomycin D dissolved in DMSO. Cell growth was determined by the MTT assay, as described in Materials and methods. Data are expressed as percent decrease of the respective untreated controls and are mean \pm SEM obtained from four independent experiments.

To determine the localization of GRP94 protein in tissues, immunohistochemistry was performed in 20 normal pancreatic tissue samples, 20 chronic pancreatitis samples, and 44 pancreatic cancer samples. In normal pancreas (Fig. 2A-C) and chronic pancreatitis (Fig. 2D-F) tissues, GRP94 was absent or weakly present in ductal cells and strongly present in acinar cells. In pancreatic cancer tissues, strong staining was detected in $20 \%$ of sections, while in $32 \%$ there was moderate staining and in $48 \%$ no staining (Fig. 2G-I). GRP94 expression was also detected in the stellate cells of tumor tissues and to some extent in stellate cells of chronic pancreatitis tissues.

Expression of GRP94 in pancreatic cancer cell lines. Next we quantified GRP94 mRNA and protein levels in eight human pancreatic cancer cell lines (Aspc-1, BxPc-3, Capan-1, Colo-357, MiaPaCa-2, Panc-1, SU86.86 and T3M4). All cancer cells analyzed expressed various degrees of GRP94 mRNA and protein (Fig. 3A).

Effects of GRP94 silencing on chemoresponsiveness of pancreatic cancer cells. Transfection of Panc-1 cells with
GRP94 siRNA for 24-120 h reduced GRP94 expression significantly in a time-dependent manner (Fig. 3B). Silencing of GRP94 had no significant effect on cell growth (data not shown).

Next, actinomycin D, which is known to induce apoptosis in pancreatic cancer cells (21), was used to induce apoptosis. Panc-1 cells were treated with GRP94 siRNA or negative control siRNA for $48 \mathrm{~h}$ before treatment with actinomycin D. After $24 \mathrm{~h}$ of incubation (72 h after transfection) with actinomycin D, there was a dose-dependent decrease in cell number, with a significant decrease of $45 \%$ in GRP94 siRNA-treated cells and only a $29 \%$ decrease in control cells $(\mathrm{p}=0.03)$ at a concentration of $100 \mathrm{ng} / \mathrm{ml}$ actinomycin D as well as at a concentration of $1000 \mathrm{ng} / \mathrm{ml}$ actinomycin D (64 vs. 50\%, $\mathrm{p}=0.03)($ Fig. 3C).

Correlation of GRP94 protein expression and patient survival. When the patients were categorized according to the staining scores, patients with strong staining (scores of 6 and 9) showed a tendency toward worse survival (19 vs. 16 months, $\mathrm{p}=0.24$ ) (Fig. 4A). There were only 3 patients with a score of 9 . Interestingly, these patients had a median survival of 


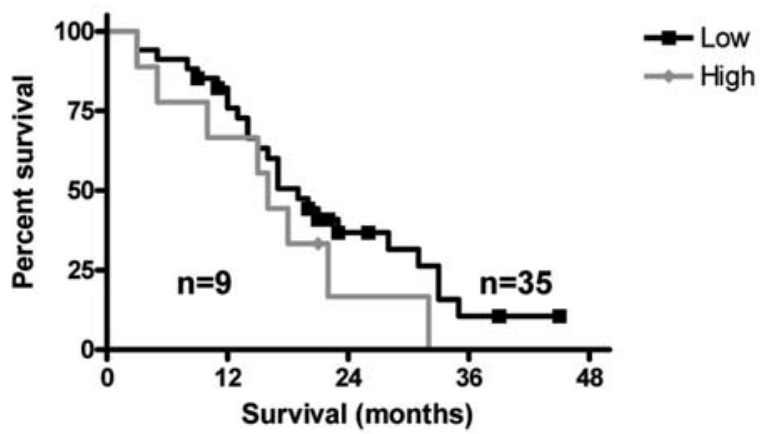

A

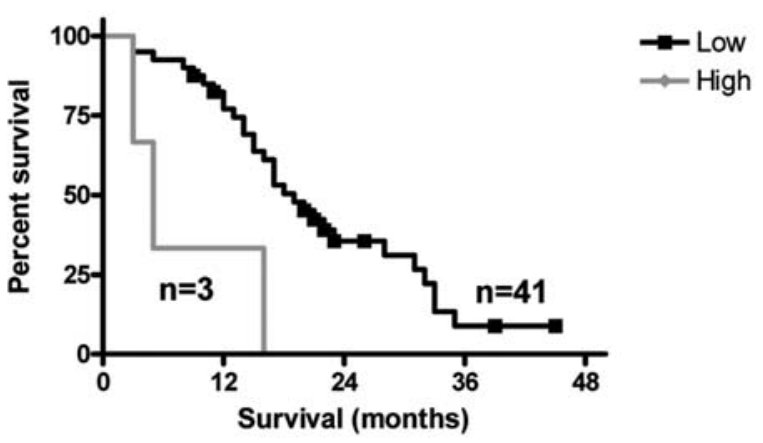

B

Figure 4. Correlation of GRP94 immunopositivity with survival in pancreatic cancer patients. Semi-quantitative immunohistochemistry was used to evaluate GRP94 immunopositivity in PDAC tissues. Scores were given separately for the stained area and for the intensity of staining. Quantification was made as follows: $1,<33 \%$ of the cancer cells; $2,33 \%-66 \%$ of the cells; $3,>66 \%$ of the cancer cells. Intensity of staining was quantified as follows: 1, absent; 2, weak/moderate; 3, strong. Each section had a final grade that was derived from the multiplication of the area and intensity scores. GRP94 expression was considered to be absent/low for grade 1, moderate for grades 2, 3, and 4, and strong for grades 6 and 9. (A) Comparison of survival in patients with low/absent and moderate $(n=35)$ vs. high $(n=9)$ IHC scores was made using the Kaplan-Meier method and log-rank analysis. (B) A subgroup analysis of survival for patients with a score of $9(n=3)$ vs. all other patients.

5 months $(\mathrm{p}=0.006)$. However, due to the small number of patients, the data should be carefully interpreted (Fig. 4B).

\section{Discussion}

Our results show that there is a significant reduction of GRP94 expression in pancreatic ductal adenocarcinoma patients. This finding is contradictory to several reports in the literature showing increased GRP94 expression in a variety of malignant tumors or cancer cell lines, such as breast (23), oral (24), lung (25), gastric (26), esophageal (27) and colonic carcinomas (28). Using immunohistochemistry we observed that GRP94 was absent or weakly present in ductal cells but strongly present in acinar cells in chronic pancreatitis and normal pancreatic tissues. This reduction in cancer can partially be explained by the loss of acinar component in tumorous areas. However, the mRNA levels of GRP94 in PDAC was also significantly lower than those of chronic pancreatitis tissues, therefore it is likely that there are other mechanisms involved in the down-regulation of GRP94 in PDAC.
Although GRP94 expression was lost in $48 \%$ of cancer tissues, it was strongly present in $20 \%$ of the cases. Low or absent expression of GRP94 showed a tendency toward better survival, but the difference was not significant in our group of 44 patients.

Recently, the endoplasmic reticulum stress pathways and the GRPs have been linked to cancer growth and drug resistance. They represent novel markers for cancer progression and chemo-responsiveness, as well as targets for cancer therapy. Previous studies have shown that overexpression of GRP94 is associated with cellular transformation, tumorigenicity and decreased sensitivity to radiation, whereas suppression of GRP94 sensitizes cells to etoposide treatment. Although several studies strongly suggest that GRP78 plays an important role in tumor growth and tumor metastasis $(29,30)$, GRP94 is still less well characterized in these aspects. Here, cell growth assays were performed to observe the effects of GRP94 silencing on cell growth. Our results show that down-regulation of GRP94 did not have a significant effect on Panc-1 pancreatic cancer cell growth.

We also examined the effects of GRP94 on apoptosis induced by treatment with actinomycin D. Actinomycin D has been reported to cause apoptosis in Panc-1 cells in conjunction with JNK/SAPK activation and enhanced expression of Bax (21). Our data demonstrate that silencing GRP94 increases the cell death induced by actinomycin D in Panc- 1 cells. The mechanisms of the antiapoptotic effect of GRP94 are not known. However, previous reports have indicated that GRP94 reduces cell death in SH-SY5Y cells, perturbs calcium homeostasis, and suppresses ischemic neuronal cell death induced by ischemia/reperfusion injury $(19,31)$. Moreover, honokiol-induced calpain-II-mediated GRP94 cleavage causes human gastric cancer cell apoptosis (32). It has also been shown that GRP94, with its calcium-binding and antiapoptotic properties, is a proteolytic target of calpain during etoposide-induced apoptosis (18).

Since all patients in the study received postoperative chemotherapy, our results show that GRP94 plays only a partial role in resistance to apoptosis in pancreatic cancer, as the tendency toward better survival in patients with absent/low expression in cancer cells did not reach statistical significance. Similarly, in esophageal cancer the level of expression of GRP94 had no correlation with clinicopathologic parameters (33). One possible explanation could be the dual function of GRP94. On the one hand, GRP94 is involved in apoptosis resistance (18-20). On the other hand, GRP94 has a significant role in promoting immunity against tumors. GRP94 may bind tumor antigens in the ER (12) which are captured during cell lysis by antigen-presenting cells and cross-primes cytotoxic CD8 ${ }^{+} \mathrm{T}$ cells $(33,34)$. Alternatively, release of GRP94 from the ER of stressed tumor cells activates the innate immune system through Toll-like receptors on antigen-presenting cells (11). Therefore, paradoxical to its effect in creating apoptosis resistance, its absence may help tumor cells to escape from immune surveillance (8).

In conclusion, our current data show that GRP94 is lost in a significant fraction of pancreatic cancer tissues, and that it might be involved in the apoptosis resistance of pancreatic cancer cells. Further studies are warranted to explore the role of GRP94 in tumor immunity in PDAC. 


\section{References}

1. Van Cutsem E, Aerts R, Haustermans K, Topal B, Van Steenbergen W and Verslype C: Systemic treatment of pancreatic cancer. Eur J Gastroenterol Hepatol 16: 265-274, 2004.

2. Erkan M, Kleeff J, Gorbachevski A, et al: Periostin creates a tumor-supportive microenvironment in the pancreas by sustaining fibrogenic stellate cell activity. Gastroenterology 132: 1447-1464, 2007.

3. Erkan M, Michalski CW, Rieder S, et al: The activated stroma index is a novel and independent prognostic marker in pancreatic ductal adenocarcinoma. Clin Gastroenterol Hepatol 6: 1155-1161, 2008.

4. Michalski CW, Kleeff J, Bachmann J, et al: Second-look operation for unresectable pancreatic ductal adenocarcinoma at a high-volume center. Ann Surg Oncol 15: 186-192, 2008.

5. Erkan M, Kleeff J, Esposito I, et al: Loss of BNIP3 expression is a late event in pancreatic cancer contributing to chemoresistance and worsened prognosis. Oncogene 24: 4421-4432, 2005.

6. Michalski CW, Erkan M, Sauliunaite D, et al: Ex vivo chemosensitivity testing and gene expression profiling predict response towards adjuvant gemcitabine treatment in pancreatic cancer. $\mathrm{Br}$ J Cancer 99: 760-767, 2008

7. Hanahan D and Weinberg RA: The hallmarks of cancer. Cell 100: 57-70, 2000.

8. Fu Y and Lee AS: Glucose regulated proteins in cancer progression, drug resistance and immunotherapy. Cancer Biol Ther 5: $741-744,2006$.

9. Koumenis C: ER stress, hypoxia tolerance and tumor progression. Curr Mol Med 6: 55-69, 2006.

10. Li J and Lee AS: Stress induction of GRP78/BiP and its role in cancer. Curr Mol Med 6: 45-54, 2006.

11. Nicchitta CV: Re-evaluating the role of heat-shock proteinpeptide interactions in tumour immunity. Nat Rev Immunol 3: 427-432, 2003

12. Srivastava PK: Therapeutic cancer vaccines. Curr Opin Immunol 18: 201-205, 2006

13. Wang XY, Li Y, Yang G and Subjeck JR: Current ideas about applications of heat shock proteins in vaccine design and immunotherapy. Int J Hyperthermia 21: 717-722, 2005.

14. Melnick J, Dul JL and Argon Y: Sequential interaction of the chaperones BiP and GRP94 with immunoglobulin chains in the endoplasmic reticulum. Nature 370: 373-375, 1994

15. Banerjea A, Ahmed S, Hands RE, et al: Colorectal cancers with microsatellite instability display mRNA expression signatures characteristic of increased immunogenicity. Mol Cancer 3: 21, 2004.

16. Kubota H, Suzuki T, Lu J, et al: Increased expression of GRP94 protein is associated with decreased sensitivity to X-rays in cervical cancer cell lines. Int J Radiat Biol 81: 701-709, 2005.

17. Lee AS: The glucose-regulated proteins: stress induction and clinical applications. Trends Biochem Sci 26: 504-510, 2001

18. Reddy RK, Lu J and Lee AS: The endoplasmic reticulum chaperone glycoprotein GRP94 with $\mathrm{Ca}(2+)$-binding and antiapoptotic properties is a novel proteolytic target of calpain during etoposide-induced apoptosis. J Biol Chem 274: 28476-28483, 1999.
19. Bando Y, Katayama T, Aleshin AN, Manabe T and Tohyama M: GRP94 reduces cell death in SH-SY5Y cells perturbated calcium homeostasis. Apoptosis 9: 501-508, 2004.

20. Lee SH, Song R, Lee MN, et al: A molecular chaperone glucose-regulated protein 94 blocks apoptosis induced by virus infection. Hepatology 47: 854-866, 2008.

21. Kleeff J, Kornmann M, Sawhney H and Korc M: Actinomycin $\mathrm{D}$ induces apoptosis and inhibits growth of pancreatic cancer cells. Int J Cancer 86: 399-407, 2000.

22. Reiser-Erkan C, Erkan M, Pan Z, et al: Hypoxia-inducible proto-oncogene Pim-1 is a prognostic marker in pancreatic ductal adenocarcinoma. Cancer Biol Ther 7: 1352-1359, 2008.

23. Gazit G, Lu J and Lee AS: De-regulation of GRP stress protein expression in human breast cancer cell lines. Breast Cancer Res Treat 54: 135-146, 1999.

24. Nomura H, Uzawa K, Yamano Y, et al: Network-based analysis of calcium-binding protein genes identifies Grp94 as a target in human oral carcinogenesis. Br J Cancer 97: 792-801, 2007.

25. Wang Q, He Z, Zhang J, et al: Overexpression of endoplasmic reticulum molecular chaperone GRP94 and GRP78 in human lung cancer tissues and its significance. Cancer Detect Prev 29: 544-551, 2005.

26. Zheng HC, Takahashi H, Li XH, et al: Overexpression of GRP78 and GRP94 are markers for aggressive behavior and poor prognosis in gastric carcinomas. Hum Pathol 39: 1042-1049, 2008.

27. Wang XP, Liu GZ, Song AL, Chen RF, Li HY and Liu Y: Expression and significance of heat shock protein 70 and glucose-regulated protein 94 in human esophageal carcinoma. World J Gastroenterol 11: 429-432, 2005.

28. Wang XP, Qiu FR, Liu GZ and Chen RF: Correlation between clinicopathology and expression of heat shock protein 70 and glucose-regulated protein 94 in human colonic adenocarcinoma. World J Gastroenterol 11: 1056-1059, 2005.

29. Misra UK, Deedwania R and Pizzo SV: Binding of activated alpha2-macroglobulin to its cell surface receptor GRP78 in 1-LN prostate cancer cells regulates PAK-2-dependent activation of LIMK. J Biol Chem 280: 26278-26286, 2005.

30. Misra UK, Deedwania R and Pizzo SV: Activation and crosstalk between Akt, NF-kappaB, and unfolded protein response signaling in 1-LN prostate cancer cells consequent to ligation of cell surface-associated GRP78. J Biol Chem 281: 13694-13707, 2006.

31. Bando Y, Katayama T, Kasai K, Taniguchi M, Tamatani M and Tohyama M: GRP94 (94 kDa glucose-regulated protein) suppresses ischemic neuronal cell death against ischemia/ reperfusion injury. Eur J Neurosci 18: 829-840, 2003.

32. Sheu ML, Liu SH and Lan KH: Honokiol induces calpainmediated glucose-regulated protein-94 cleavage and apoptosis in human gastric cancer cells and reduces tumor growth. PLoS ONE 2: e1096, 2007

33. Parmiani G, Testori A, Maio M, et al: Heat shock proteins and their use as anticancer vaccines. Clin Cancer Res 10: 8142-8146, 2004.

34. Wang XH, Qin Y, Hu MH and Xie Y: Dendritic cells pulsed with gp96-peptide complexes derived from human hepatocellular carcinoma (HCC) induce specific cytotoxic T lymphocytes. Cancer Immunol Immunother 54: 971-980, 2005. 